\title{
Simultaneous co-ingestion of prescription stimulants, alcohol and other drugs: a multi-cohort national study of US adolescents
}

\author{
Sean Esteban McCabe ${ }^{1,2}$, Brady T. West ${ }^{3,4}$, Ty S. Schepis ${ }^{5}$ and Christian J. Teter ${ }^{6}$ \\ ${ }^{1}$ Institute for Research on Women and Gender, University of Michigan, Ann Arbor, MI, USA \\ ${ }^{2}$ Substance Abuse Research Center, University of Michigan, Ann Arbor, MI, USA \\ ${ }^{3}$ Center for Statistical Consultation and Research, University of Michigan, Ann Arbor, MI, USA \\ ${ }^{4}$ Survey Research Center, Institute for Social Research, University of Michigan, Ann Arbor, MI, USA \\ ${ }^{5}$ Department of Psychology, Texas State University, San Marcos, TX, USA \\ ${ }^{6}$ College of Pharmacy, University of New England, Portland, ME, USA
}

\begin{abstract}
Objective To determine the past-year prevalence rates and correlates of simultaneous co-ingestion of prescription stimulants and other substances among US high school seniors.

Methods Nationally representative probability samples of US high school seniors were surveyed as a part of the Monitoring the Future study. The sample consisted of five cohorts including a total of 12431 high school seniors (modal age: 18 years) and represented a population that was $53 \%$ female.

Results Among past-year nonmedical users of prescription stimulants $(n=835)$, the estimated prevalence of any past-year simultaneous coingestion of prescription stimulants and other substances was $64.4 \%$. The substances most commonly co-ingested with prescription stimulants included marijuana $(51.1 \%)$ and alcohol (48.4\%). Nonmedical users who co-ingested prescription stimulants with other substances were more likely to report non-oral routes of administration, recreational motives and greater subjective high when using prescription stimulants than nonmedical users who did not co-ingest prescription stimulants with other substances.

Conclusions The majority of past-year nonmedical users of prescription stimulants reported simultaneous co-ingestion of prescription stimulants and other substances. The findings indicate that co-ingestion of prescription stimulants and other substances is a pervasive behavior among US adolescents who engage in nonmedical use of prescription stimulants and should be carefully considered in future clinical practice and research. Copyright (C) 2014 John Wiley \& Sons, Ltd.
\end{abstract}

KEY WORDS—-prescription stimulants; co-ingestion; simultaneous use; adolescents; polydrug use

\section{INTRODUCTION}

The nonmedical use of prescription stimulants and stimulant use disorders are most prevalent among adolescents and young adults in the US, although these behaviors have increased across all age groups over the past two decades (Blanco et al., 2007; McCabe et al., 2008; Johnston et al., 2013). For example, the past-year nonmedical use of prescription stimulants increased from $3.6 \%$ in 1992 to $11.1 \%$ in 2012 among college students in the US (Johnston et al., 2013). National, regional and case-report data document a wide range of adverse consequences that can occur as a result of simultaneous co-ingestion of prescription stimulants with alcohol and other drugs

*Correspondence to: S. E. McCabe, Institute for Research on Women and Gender, University of Michigan, 204 S. State St., Ann Arbor, MI 48109-1290, USA. Tel: (734) 615-8840; Fax: (734) 615-2931 E-mail: plius@umich.edu
(Markowitz et al., 2000a, 2000b; Barrett and Pihl, 2002; Watson et al., 2004; McCabe et al., 2006; SAMHSA, 2013a, 2013b). Data from the Drug Abuse Warning Network indicate that emergency department (ED) visits associated with nonmedical use of attention-deficit/hyperactivity disorder (ADHD) stimulant medications often involve the simultaneous use of other substances (SAMHSA, 2013a, 2013b). Indeed, marijuana was the most common substance simultaneously co-ingested with ADHD stimulant medications among adolescents aged 15 to 17 years involved in ED visits, while alcohol was the most common substance simultaneously co-ingested with ADHD stimulant medications among young adults aged 18 to 25 years involved in ED visits (SAMHSA, $2013 \mathrm{~b})$. The estimated number of ED visits involving the nonmedical use of prescription stimulants has steadily increased among those 18 to 25 years of age (SAMHSA, 2013a, 2013b). For example, the number 
of ED visits involving ADHD stimulant medications increased from 2131 in 2005 to 8148 in 2010 , or $382 \%$ (SAMHSA, 2013b).

The simultaneous co-ingestion of prescription medications and other psychoactive drugs is a behavior that has been shown to increase the risk for substance use disorders (Compton and Volkow, 2006; McCabe et al., 2006). Despite the notable risks associated with co-ingestion of prescription stimulants and other psychoactive drugs, there is surprisingly little epidemiological research directed at the prevalence of and characteristics associated with the co-ingestion of nonmedical use of prescription stimulants and other drugs among adolescents (Collins et al., 1998; Compton and Volkow, 2006; Earleywine and Newcomb, 1997). A few college-based studies have shown that the majority of nonmedical users of prescription stimulants have co-ingested prescription stimulants and other psychoactive drugs (Barrett et al., 2005; McCabe et al., 2006; Olthuis et al., 2013). To date, there have been no national epidemiological studies that examine co-ingestion of prescription stimulants and other drugs among secondary school students. Based on these gaps in the existing literature, the main objectives of this study were to identify the prevalence and correlates of past-year co-ingestion of prescription stimulants and other drugs in a national sample of high school seniors in the US.

\section{METHODS}

\section{Study design}

The Monitoring the Future (MTF) study annually surveys a cross-sectional, nationally representative sample of high school seniors in approximately 135 public and private high schools in the coterminous US (Johnston et al., 2013). The MTF study uses a multi-stage sampling procedure: In stage 1, geographic areas or primary sampling units are selected; in stage 2, schools within primary sampling units are selected (with probability proportionate to class size); and in stage 3, students within schools are selected. Because so many questions are included in the MTF study, much of the questionnaire content is divided into six different questionnaire forms that are randomly distributed. This approach results in six virtually identical subsamples. The data collected from seniors receiving Form 1 from 2002 to 2006 were used in this study because these MTF surveys contained the most recent questions regarding co-ingestion of prescription stimulants and other substances in a national sample. The student response rates for high school seniors ranged from $82 \%$ to $83 \%$ for these cohorts. The school participation rates ranged from $97 \%$ to $99 \%$ for these cohorts for either originally sampled schools or replacement schools. Additional details about the MTF design and methods are available elsewhere (Johnston et al., 2013). Approval was granted for this study by the University of Michigan Institutional Review Board Health Sciences.

\section{Measures}

The MTF study assesses demographic characteristics such as sex, race, geographical region and substance use behaviors.

Nonmedical use of prescription stimulants was assessed with a series of items asking respondents on how many occasions (if any) they used prescription stimulants on their own, without a doctor's orders (e.g., Ritalin ${ }^{\circledR}$ and Dexedrine ${ }^{\circledR}$ ). Respondents were asked about nonmedical use of prescription stimulants in the past 12 months. The response scale ranged from (1) no occasions to (7) 40 or more occasions.

Simultaneous co-ingestion of prescription stimulants and other substances was measured with four items focused on the number of times prescription stimulants were used nonmedically at the same time as the following substances so that the effects overlapped: alcohol, marijuana, LSD and hallucinogens other than LSD. The response scale ranged from (1) not at all to (5) every time for each of the four items.

Drunkenness was measured using the following item: "On how many occasions (if any) have you been drunk or very high from drinking alcoholic beverages during the last 12 months?" The response scale was the same as for nonmedical use of prescription stimulants.

Cigarette use was measured using the following item: "How frequently have you smoked cigarettes during the past 30 days?" The response scale was (1) none, (2) less than 1 cigarette per day, (3) 1-5 cigarettes per day, (4) about 1/2 pack per day, (5) about 1 pack per day, (6) about 11/2 packs per day and (7) 2 or more packs per day.

Marijuana use was measured using the following item: "On how many occasions (if any) have you used marijuana during the last 12 months?" The response scale was the same as for nonmedical use of prescription stimulants.

Other drug use-including LSD, psychedelics other than LSD, cocaine and heroin-was measured with the following question for each drug: "On how many occasions (if any) have you used [SPECIFIED DRUG CLASS]...during the last 12 months?" The response 
scale was the same as for nonmedical use of prescription stimulants.

Nonmedical use of other prescription medicationsincluding opioids, sedatives and tranquilizers - was measured with the following question for each medication class: "On how many occasions (if any) have you used [SPECIFIED DRUG CLASS]...during the last 12 months?" The response scale was the same as for nonmedical use of prescription stimulants.

Routes of administration for nonmedical use of prescription stimulants were assessed with four items that asked which methods respondents used for taking prescription stimulants (mark all that apply). The binary items included the following: (1) smoking; (2) injection; (3) orally (by mouth); and (4) other.

Social contexts of nonmedical use of prescription stimulants were assessed by asking respondents the locations they used prescription stimulants. The list of locations included but was not limited to the following: (1) at a party.

Motives for nonmedical use of prescription stimulants were assessed by asking respondents who reported nonmedical use to indicate the most important reasons for nonmedical use (mark all that apply). The list of binary items included but was not limited to the following: (1) to feel good or get high; (2) to increase the effects of some other drugs; and (3) to decrease (offset) the effects of some other drugs.

Subjective high associated with nonmedical use of prescription stimulants was measured with an item that asked nonmedical users in the past 12 months how high they usually get when they use prescription stimulants. The response scale for these items ranged from (1) not at all high to (4) very high.

\section{Data analysis}

The MTF study provides survey weights for responding cases in each of its public-use data files, and these weights were used in all analyses to ensure that estimates of population features were unbiased. The estimated past-year prevalence rates of co-ingestion involving nonmedical use of prescription stimulants and other drugs - across subgroups defined by demographic characteristics and substance use behaviorswere computed using weighted cross-tabulations. Design-adjusted Rao-Scott chi-square tests of homogeneity (Rao and Scott, 1984) and logistic regression analyses (Heeringa et al., 2010) were conducted to determine whether past-year simultaneous co-ingestion involving nonmedical use of prescription stimulants and other drugs was significantly associated with other substance use behaviors, including past-year drunkenness, smoking in the past 30 days, past-year marijuana use, past-year illicit drug use other than marijuana and past-year nonmedical use of other prescription medications such as opioids, sedatives and tranquilizers. The following three mutually exclusive groups were compared in terms of other substance use behaviors in the analyses: (1) no past-year nonmedical use of prescription stimulants; (2) past-year nonmedical use of prescription stimulants without simultaneous co-ingestion; and (3) past-year nonmedical use of prescription stimulants with simultaneous co-ingestion. The logistic regression models included sex, race/ethnicity, geographical region, average grades, college intentions and frequency of nonmedical use of prescription stimulants (Tables 4 and 5 only) as covariates based on their significant bivariate associations with dependent and independent variables used in the present study (e.g., the three-group factor discussed earlier), nonmedical use of prescription stimulants, and/or co-ingestion of prescription stimulants and other drugs (McCabe et al., 2006; Johnston et al., 2013; Chen et al., 2014).

The complex multi-stage sampling design used in the MTF study resulted in the need to account for effects of cluster sampling on variance estimates. Estimated (linearized) variances of weighted estimates were multiplied by an average MTF design effect factor corrected for design effects because of the cluster sampling prior to the construction of confidence intervals, and weighted Pearson chi-square statistics were divided by this same design effect factor (Rao and Scott, 1984) per the recommendation of Johnston and colleagues (Johnston et al., 2013). All statistical analyses were performed using commands for the analysis of complex sample survey data in the Stata 13.1 software (StataCorp, College Station, TX, 2013).

\section{RESULTS}

\section{Sample characteristics}

There were 12431 individuals who completed Form 1 in the five cohorts between 2002 and 2006 during the spring of their senior year, and these respondents comprise the study sample. The full sample represented a population of high school seniors that was $52.7 \%$ women, $61.8 \%$ White, $10.1 \%$ AfricanAmerican, and $28.1 \%$ from other racial groups or not specifying their race. The modal age of the individuals in the sample was 18 years of age. Table 1 compares the sociodemographic characteristics between the subpopulations of past-year nonmedical users of prescription stimulants who coingested other substances and past-year nonmedical 
CO-INGESTION OF PRESCRIPTION STIMULANTS AND OTHER DRUGS

Table 1. Demographic characteristics among high school seniors in the US: past-year nonmedical users of prescription stimulants versus overall population

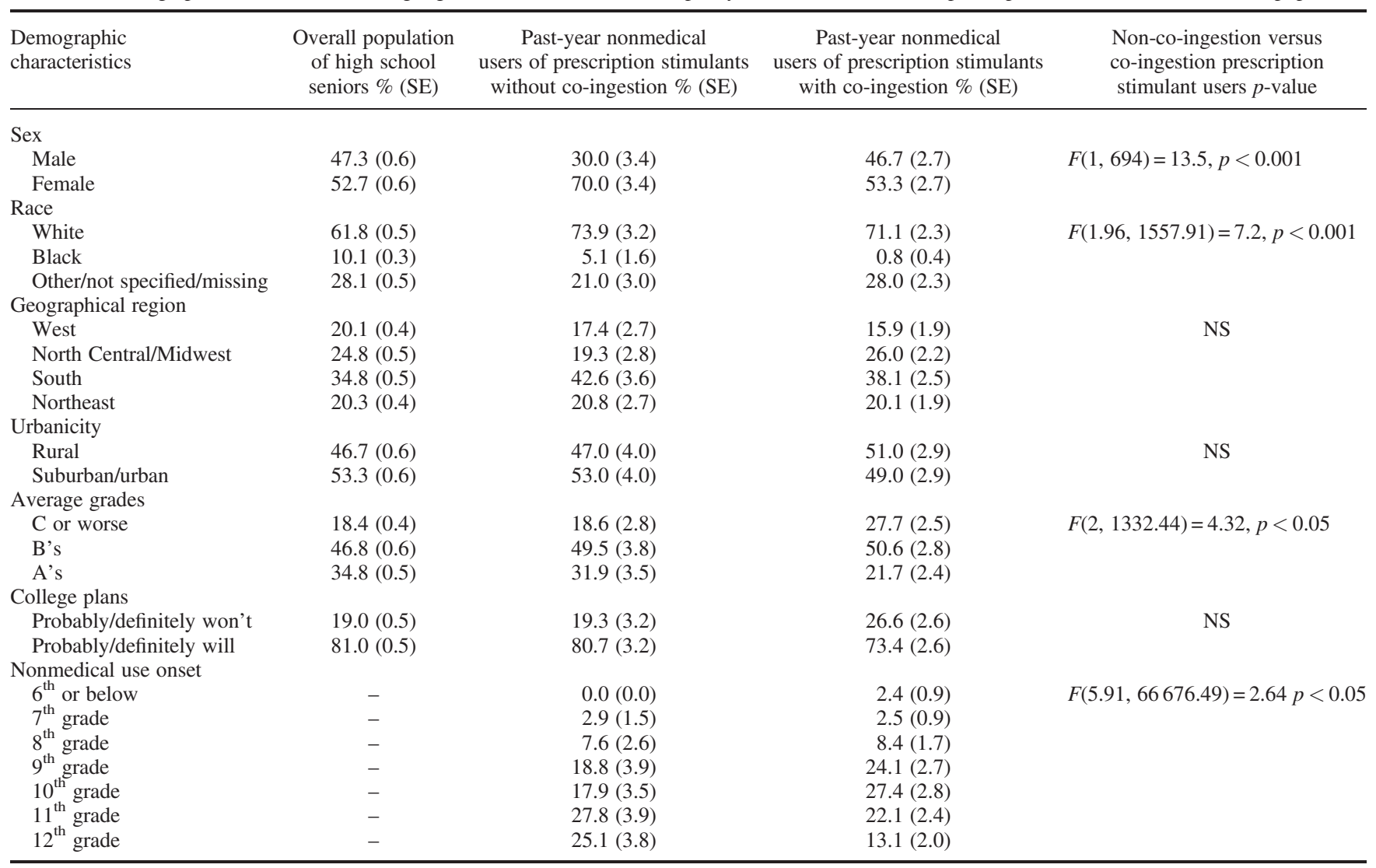

NS, non-significant at the 0.05 level; -, not applicable.

users of prescription stimulants who did not co-ingest other substances.

\section{Simultaneous co-ingestion of prescription stimulants and other drugs}

The estimated prevalence of lifetime nonmedical use of prescription stimulants among high school seniors in the US between the years of 2002 and 2006 was $11.2 \%(\mathrm{SE}=0.3 \%)$, while the prevalence of past-year nonmedical use of prescription stimulants was $7.3 \%(\mathrm{SE}=0.3 \%)$. Among high school seniors who reported past-year nonmedical use of prescription stimulants $(n=835)$, an estimated $34.0 \%$ used on 1 to 2 occasions, $20.5 \%$ used on 3 to 5 occasions, $13.6 \%$ used on 6 to 9 occasions and $31.9 \%$ used on 10 or more occasions. The majority of past-year nonmedical users of prescription stimulants simultaneously co-ingested at least one other substance while using prescription stimulants. Indeed, the estimated prevalence of any past-year simultaneous co-ingestion of prescription stimulants and other substances (i.e., alcohol, marijuana, LSD and other psychedelics) among past-year nonmedical prescription stimulant users was $64.3 \% \quad(\mathrm{SE}=2.0 \%$, $95 \% \mathrm{CI}=60.4 \%, 68.3 \%$ ). Among past-year nonmedical users of prescription stimulants, the prevalence of co-ingestion differed significantly by race/ethnicity $(p<0.001)$, and a higher proportion of past-year nonmedical users who also co-ingested was found to be male compared with those who did not co-ingest in the past year (Table 1). Significant $(p<0.05)$ associations were also found between co-ingestion and average grade point average as well as nonmedical stimulant use age of onset; those who co-ingested in the past-year tended to have lower average grades and earlier ages of onset.

The most prevalent forms of simultaneous coingestion of prescription stimulants and other substances included marijuana $(51.2 \%)$, alcohol $(48.7 \%)$ and non-LSD hallucinogens $(9.2 \%)$. We examined the estimated frequency of co-ingestion of prescription stimulants and other substances among nonmedical users of prescription stimulants (Table 2). Among those past-year nonmedical users who co-ingested prescription stimulants with other substances, we found that co-ingestion was more likely to occur "a few times" 
Table 2. Co-ingestion of prescription stimulants and other substances among past-year nonmedical prescription stimulant users

\begin{tabular}{lccrrr}
\hline Co-ingested substances $(n)^{\text {a }}$ & Not at all \% (SE) & A few times \% (SE) & Sometimes \% (SE) & Most times \% (SE) & Every time \% (SE) \\
\hline Marijuana $(n=771)$ & $48.8(2.1)$ & $16.9(1.5)$ & $15.0(1.5)$ & $9.5(1.2)$ & $9.9(1.2)$ \\
Alcohol $(n=773)$ & $51.3(2.1)$ & $21.1(1.7)$ & $15.2(1.5)$ & $5.8(0.9)$ & $6.6(1.0)$ \\
Hallucinogens other than LSD $(n=679)$ & $90.8(1.2)$ & $3.9(0.7)$ & $2.7(0.7)$ & $1.8(0.6)$ & $0.9(0.3)$ \\
LSD $(n=753)$ & $94.4(1.0)$ & $1.7(0.5)$ & $1.5(0.5)$ & $1.8(0.7)$ & $0.7(0.3)$ \\
\hline
\end{tabular}

${ }^{a}$ The $(n)$ refers to the count of past-year nonmedical users of stimulants with valid data on each co-ingestion indicator.

or "sometimes" as compared with "most times" or "every time." Among those past-year nonmedical users who co-ingested prescription stimulants with other substances and provided valid responses to all of the co-ingestion questions, an estimated $42.4 \%$ reported co-ingestion of one other substance, while the majority $(57.6 \%)$ reported co-ingestion of two or more substances.

For past-year nonmedical prescription stimulant users, we found that simultaneous co-ingestion of any other substances was more prevalent among frequent nonmedical users of prescription stimulants (10 or more occasions in the past year) than nonmedical users who used less frequently (less than 10 occasions in the past year) $(70.0 \%$ vs. $61.7 \%$; $F(1,11511)=3.71, p=0.05)$. For past-year marijuana users, we found that simultaneous co-ingestion of marijuana and prescription stimulants was more prevalent among frequent marijuana users (10 or more occasions in the past year) than marijuana users who used less frequently (less than 10 occasions in the past year) $(76.8 \%$ vs. $21.0 \% ; F(1,8357)=139.73$, $p<0.01)$. For past-year alcohol users, we found that simultaneous co-ingestion of alcohol and prescription stimulants was more prevalent among frequent alcohol users (10 or more occasions in the past year) than alcohol users who used less frequently (less than 10 occasions in the past year) $(60.5 \%$ vs. $25.7 \%$; $F(1,4024)=57.14, p<0.01)$.

\section{Simultaneous co-ingestion and other substance use behaviors}

The chi-square analyses revealed significant associations between simultaneous co-ingestion of prescription stimulants and other drugs and each substance use behavior $(p<0.001)$. As illustrated in Table 3, multivariate logistic regression results reinforced the bivariate findings; after adjusting for covariates found to be significantly associated with co-ingestion (sex, race, geographical region, grade point average and college plans), the odds of reporting substance use behaviors were considerably higher among individuals who reported past-year nonmedical use of prescription stimulants (both with and without co-ingestion) compared with those who did not engage in past-year nonmedical use of prescription stimulants $(p<0.001)$. In particular, the odds of reporting substance use behaviors were substantially higher for those who also reported co-ingestion.

\section{Simultaneous co-ingestion and specific behaviors related to prescription stimulants}

The associations among simultaneous co-ingestion of nonmedical use of prescription stimulants and other drugs and specific behaviors related to the use of prescription stimulants, such as route of administration, social context, motives and subjective high, were also examined using design-adjusted chi-square analyses, revealing several strongly significant associations $(p<0.001)$. As illustrated in Table 4, multiple logistic regression results largely supported the bivariate findings; the odds of non-oral administration, using stimulants at a party, using stimulants to get high or feel good, using stimulants to increase or decrease the effects of other drugs and using stimulants to get moderately or very high were all significantly greater among those nonmedical users who co-ingested prescription stimulants with other drugs as compared with the odds of these behaviors for those nonmedical users who did not report co-ingestion, after adjusting for frequency of nonmedical use, sex, race, geographic region, grade point average and college plans $(p<0.001)$. Notably, the odds of using prescription stimulants to get high or feel good were more than 3.9 times greater among those nonmedical users who co-ingested prescription stimulants with other drugs as compared with those nonmedical users who did not report co-ingestion $(58.9 \%$ vs. $25.7 \%, \mathrm{AOR}=3.9$, $95 \% \mathrm{CI}=2.5,6.2, p<0.001)$.

We also examined the associations between the number of substances co-ingested with prescription stimulants and specific behaviors related to the use of prescription stimulants, using design-adjusted RaoScott chi-square analyses and logistic regression analyses. We found that the odds of non-oral administration, using stimulants at a party, using stimulants to get high or feel good, using stimulants to increase or decrease the effects of other drugs and using stimulants 
CO-INGESTION OF PRESCRIPTION STIMULANTS AND OTHER DRUGS
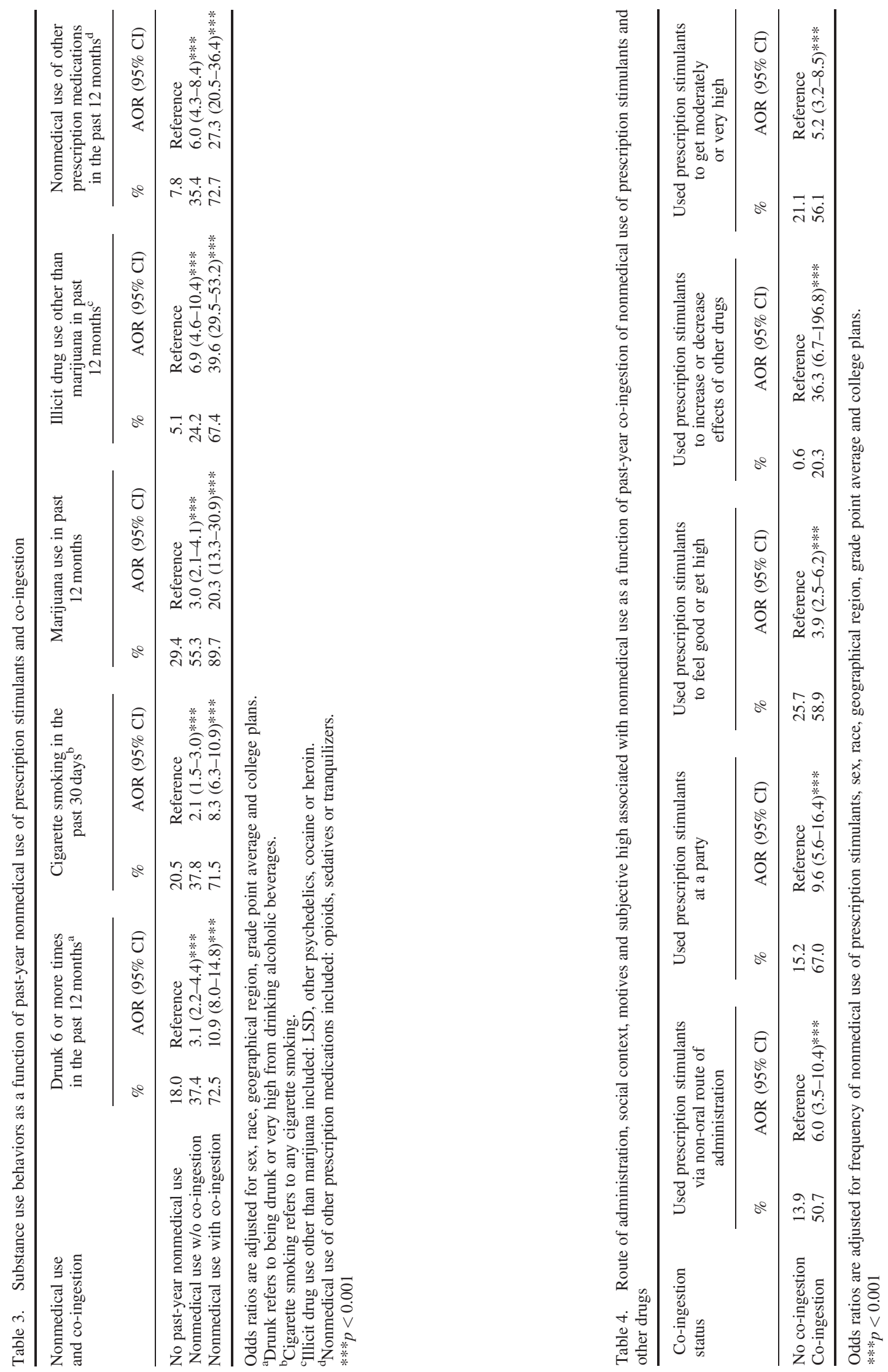
to get moderately or very high increased as a function of the number of drugs co-ingested with prescription stimulants (Table 5). For example, the odds of using stimulants at a party were nearly 16 times greater among those nonmedical users who co-ingested prescription stimulants with two or more drugs as compared with those nonmedical users who did not report co-ingestion $(\mathrm{AOR}=15.7,95 \% \mathrm{CI}=8.6,28.8$, $p<0.001$ ), after adjusting for frequency of nonmedical use, sex, race, geographic region, grade point average and college plans. Finally, we found that co-ingestion involving prescription stimulants and two or more drugs increased the odds of non-oral administration, using stimulants at a party and using stimulants to increase or decrease the effects of other drugs more than co-ingestion with one drug (results not shown).

\section{DISCUSSION}

This was the first national study to examine simultaneous co-ingestion involving prescription stimulants, alcohol and other drugs among high school seniors in the US. The findings indicate that over 6 in every 10 nonmedical stimulant users report the simultaneous co-ingestion of prescription stimulants, alcohol and other drugs in the previous year. A few prior investigations of college students found that the majority of nonmedical users of prescription stimulants reported co-ingestion of prescription stimulants and other drugs in the past year (Barrett et al., 2005; McCabe et al., provide compelling evidence, based on a national sample of high school seniors, that the majority of past-year nonmedical users of prescription stimulants have simultaneously co-ingested at least one other drug while using prescription stimulants in the past year.

We found that the most prevalent forms of simultaneous co-ingestion involving prescription stimulants included marijuana or alcohol among high school seniors in the US, which is consistent with past studies among adolescents and young adults in North America (Barrett et al., 2005; McCabe et al., 2006; Olthuis et al., 2013; SAMHSA, 2013a, 2013b). The high rates of co-ingestion involving prescription stimulants, marijuana and alcohol found among high school seniors in the present study could be partially related to the high prevalence and perceived availability of alcohol and marijuana use among adolescents (Johnston et al., 2013). For example, approximately 36\% of high school seniors have used marijuana in the past 12 months, while over $80 \%$ indicate marijuana is fairly easy or very easy to obtain (Johnston et al., 2006; Olthuis et al., 2013). The findings of this study

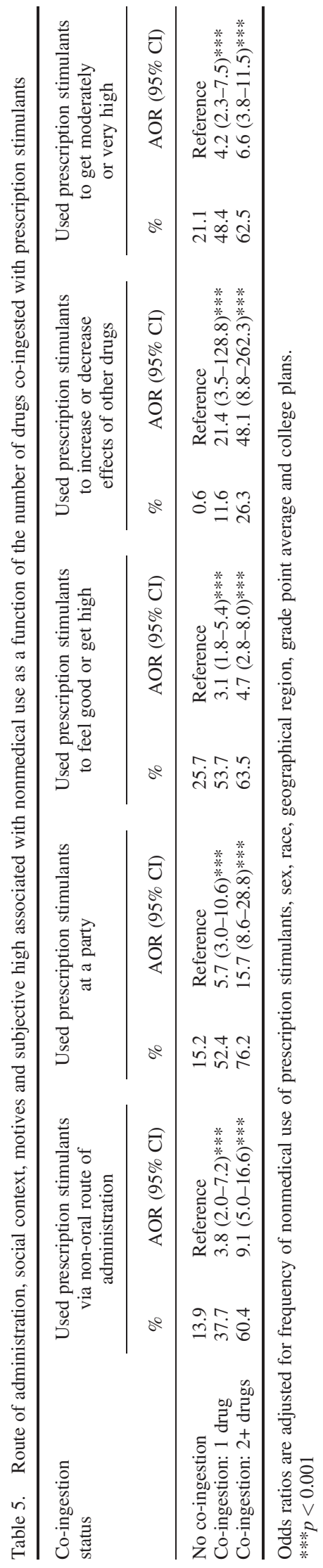

Copyright (C) 2014 John Wiley \& Sons, Ltd.
Hum. Psychopharmacol Clin Exp 2015; 30: 42-51

DOI: $10.1002 /$ hup 
2013). Similarly, approximately $64 \%$ of high school seniors have used alcohol in the past 12 months, while over $90 \%$ indicate alcohol is fairly easy or very easy to obtain (Johnston et al., 2013). The present study found that simultaneous co-ingestion was more likely to occur "a few times" or "sometimes" as compared with "most times" or "every time" among those past-year nonmedical users who co-ingested prescription stimulants with other substances. Past collegebased research found that the mean number of days of simultaneous co-ingestion of nonmedical use of prescription stimulants and alcohol was 3.3 days (range 0-30 days) in the past year (McCabe et al., 2006). Interestingly, alcohol was used in greater quantities when co-ingested with methylphenidate than when alcohol was used alone by college students (Barrett et al., 2006).

The simultaneous co-ingestion of prescription stimulants and other psychoactive drugs can result in additional reinforcement, thereby increasing the addictive potential (Compton and Volkow, 2006). Although the causal mechanism(s) explaining why marijuana is the drug most commonly co-ingested with prescription stimulants requires additional investigation, there is increasing evidence that cannabinoids modulate brain reward systems closely involved in stimulant addiction (Olière et al., 2013). A recent comprehensive review regarding the interactions between the cannabinoid system and stimulant use concluded that there are a variety of ways in which cannabinoids (endogenous and exogenous) may interact with psychostimulants (Olière et al., 2013), which in turn could help to explain their co-ingestion among users. In addition to the myriad of explanations provided by this comprehensive review, another highly relevant variable to consider regarding the high rates of co-ingestion of marijuana and stimulants is the low perceived consequences associated with marijuana use among adolescents (Johnston et al., 2013).

Regarding the substance that is the second most commonly co-ingested substance with prescription stimulants (i.e., alcohol) in this study, there is a surprising lack of research available in the literature to support or refute this finding. In fact, mechanisms to explain the common occurrence and the apparent appeal of co-ingestion of stimulants (more generally) and alcohol is currently being investigated by the National Institutes of Health (NIH). For example, there is a recent funding opportunity announcement (PA-13-339) from the NIH "...to promote research to study the neurobiological and behavioral mechanisms that might explain how alcohol and stimulants interact at genetic, epigenetic, cellular, neurocircuitry and behavioral levels to promote co-addiction." One aspect of stimulant and alcohol co-ingestion that has been described in the literature is the possibility of bidirectional vulnerability to the stimulant effects of each substance. For example, Allen and Gabbay (2013) discussed the idea that individuals who are very responsive to the stimulant effects of amphetamines may be more responsive to the stimulant effects of alcohol. In this original research report, various potential mechanisms (e.g., personality traits and reward sensitivity) are discussed, as well as a call for further research (Allen and Gabbay, 2013).

Previous research has shown a wide array of acute and long-term adverse consequences associated with the simultaneous co-ingestion of prescription stimulants and other drugs among adolescents and young adults (Watson et al., 2004; McCabe et al., 2006; SAMHSA, 2013a, 2013b). This study highlights that substance use behaviors and health risks are more prevalent among nonmedical users who co-ingest prescription stimulants and other drugs relative to nonmedical users of prescription stimulants who do not co-ingest and non-users. We found that nonmedical users who co-ingested prescription stimulants with other drugs were significantly more likely than other nonmedical users and non-users to engage in problematic substance use behaviors, even after statistically controlling for relevant covariates. Consistent with recent national findings (SAMHSA, 2013b), we found higher rates of adverse substance use behaviors (e.g., non-oral administration) as a function of the number of drugs co-ingested with prescription stimulants. Lastly, research has demonstrated that the simultaneous co-ingestion of nonmedical prescription stimulants and alcohol among college students was associated with lower grade point averages and increased substance-related consequences (McCabe et al., 2006; Egan et al., 2013). Taken together, these findings reinforce the importance of distinguishing nonmedical users who simultaneously co-ingest prescription stimulants and other drugs from other nonmedical users.

The present study extends existing knowledge by identifying several behavioral correlates associated with simultaneous co-ingestion of prescription stimulants and other drugs, such as recreational social contexts, recreational motives, subjective high, frequent nonmedical use of prescription stimulants, frequent alcohol use, frequent marijuana use and non-oral routes of administration. This study found that nearly $60 \%$ of nonmedical users who co-ingested were motivated to get high or experiment and about $70 \%$ of nonmedical users who co-ingested used at parties; these 
results suggest that motives and social contexts for coingestion are primarily recreational in nature. In addition, the majority of nonmedical users who coingested prescription stimulants and other drugs reported non-oral routes of administration when using prescription stimulants, relative to less than $14 \%$ of nonmedical users who did not co-ingest. Taken together, these behavioral correlates can potentially serve as important signals to include in screening efforts to detect nonmedical users of prescription stimulants at the highest risk for developing substance use disorders in a clinical setting. In addition, health professionals should be aware that adolescent nonmedical users of prescription stimulants are likely to be unaware of the stimulant's potential for interaction with other drugs or alternatively the drug's documented contraindications and precautions. Many adolescents also tend to underestimate the perceived harmfulness associated with the nonmedical use of prescription stimulants, and many do not understand the dangerous interactions from simultaneously co-ingesting prescription stimulants and other substances (Johnston et al., 2013). For example, passing out can be a protective mechanism that stops people from drinking when they are approaching potentially dangerous blood-alcohol concentrations. However, using prescription stimulants while drinking can potentially override this mechanism, and this could lead to life-threatening consequences.

The present study features several notable strengths, such as the inclusion of a large national sample of high school seniors. This study is the first attempt to assess simultaneous co-ingestion involving prescription stimulants and other psychoactive drugs among high school seniors in the US. Despite the strengths, there were also several limitations that should be noted when considering the implications of the findings. First, the results cannot be generalized to all adolescents because this sample only included high school seniors (modal age: 18 years) and did not include individuals who had dropped out of school or were not present in school on the day of survey administration. Second, the data are subject to the potential response bias introduced when assessing sensitive behaviors via selfreport surveys administered in a school setting. The present study attempted to minimize these biases by informing potential respondents that participation was voluntary and assuring potential respondents that data would remain confidential (Johnston and O'Malley, 1985; Harrison and Hughes, 1997). Third, because the present study represented secondary analyses, the survey items in the MTF limited what variables could be examined. For example, survey items were limited to co-ingestion involving alcohol, marijuana, LSD and hallucinogens other than LSD, and these items did not specify the dose taken at each instance of coingestion. Finally, the cross-sectional nature of the study presented some limitations in terms of making causal inferences; more comprehensive longitudinal studies are needed to examine co-ingestion of prescription stimulants and other drugs.

In summary, this study found that almost two-thirds of past-year nonmedical users of prescription stimulants simultaneously co-ingest prescription stimulants and other psychoactive drugs. The findings of this study have several important implications for prevention and intervention efforts. Nonmedical users who simultaneously co-ingest prescription stimulants and other drugs are significantly more likely than other nonmedical users and non-users to engage in problematic substance use behaviors. These findings indicate the importance of identifying nonmedical users of prescription stimulants who co-ingest prescription stimulants and other drugs. Screening efforts to detect adolescents who co-ingest prescription stimulants and other drugs at an early stage would help identify a subgroup of individuals who may benefit from a more comprehensive substance abuse assessment and treatment. Prevention programs that provide information and educate adolescents about blood-alcohol concentration levels may not be relevant when adolescents are combining prescription drugs with alcohol and other drugs. Prevention and intervention efforts should make adolescents aware of the potential consequences associated with the co-ingestion of prescription stimulants and other drugs.

\section{CONFLICT OF INTEREST}

The authors have no conflicts of interest to report.

\section{ACKNOWLEDGEMENTS}

The development of this article was supported by research grants R01DA024678, R01DA031160 and T32 DA007267 from the National Institute on Drug Abuse, National Institutes of Health. The content is solely the responsibility of the authors and does not necessarily represent the official views of the National Institute on Drug Abuse or the National Institutes of Health. The Monitoring the Future data were collected by a research grant R01DA01411 from the National Institute on Drug Abuse, National Institutes of Health. The authors would like to thank the Substance Abuse and Mental Health Data Archive for providing access to these data. 


\section{REFERENCES}

Allen KJ, Gabbay FH. 2013. The amphetamine response moderates the relationship between negative emotionality and alcohol use. Alcohol Clin Exp Res 37: 348-360.

Barrett SP, Darredeau C, Bordy LE, Pihl RO. 2005. Characteristics of methylphenidate misuse in a university student sample. Can J Psychiatry 50: 457-461.

Barrett SP, Darredeau C, Pihl RO. 2006. Patterns of simultaneous polysubstance use in drug using university students. Hum Psychopharmacol Clin Exp 21: 255-263.

Barrett SP, Pihl RO. 2002. Oral methylphenidate-alcohol co-abuse. J Clin Psychopharmacol 22: 633-634.

Blanco C, Alderson D, Ogburn E, et al. 2007. Changes in the prevalence of non-medical prescription drug use and drug use disorders in the United States: 1991-1992 and 2001-2002. Drug Alcohol Depend 90: 252-260.

Chen LY, Strain EC, Alexandre PK, Alexander GC, Mojtabai R, Martins SS. 2014. Correlates of nonmedical use of stimulants and methamphetamine use in a national sample. Addict Behav 39: 829-836.

Collins RL, Ellickson PL, Bell RM. 1998. Simultaneous polydrug use among teens: prevalence and predictors. J Subst Abuse 10: 233-253.

Compton WM, Volkow ND. 2006. Abuse of prescription drugs and the risk of addiction. Drug Alcohol Depend 83: 4-7.

Earleywine M, Newcomb MD. 1997. Concurrent versus simultaneous polydrug use: prevalence, correlates, discriminant validity, and prospective effects on health outcomes. Exp Clin Psychopharmacol 5: 353-364.

Egan KL, Reboussin BA, Blocker JN, Wolfson M, Sutfin EL. 2013. Simultaneous use of non-medical ADHD prescription stimulants and alcohol among undergraduate students. Drug Alcohol Depend 131: 71-77.

Harrison L, Hughes A. 1997. The validity of self-reported drug use: improving the accuracy of survey estimate. In NIH Publication 97-4147, NIDA Research Monograph No. 167. Government Printing Office: Washington; 1-16.

Heeringa SG, West BT, Berglund PA. 2010. Applied Survey Data Analysis. Chapman and Hall: London.

Johnston LD, O'Malley PM. 1985. Issues of validity and population coverage in student surveys of drug use. NIDA Res Monogr 57: 31-54.
Johnston LD, O’Malley PM, Bachman JG, Schulenberg JE. 2013. Monitoring the Future National Survey Results on Drug Use, 1975-2012. Volume I: Secondary School Students. University of Michigan Institute for Social Research: Ann Arbor, MI.

Markowitz JS, Logan BK, Diamond F, Patrick KS. 2000a. Detection of the novel metabolite ethylphenidate after methylphenidate overdose with alcohol coingestion. J Clin Psychopharmacology 19:362-366.

Markowitz JS, Devane CL, Boulton DW, Risch SC, Diamond F, Patrick KS. 2000b. Ethylphenidate formation in human subjects after the administration of a single dose of methylphenidate and ethanol. Drug Metab Dispos 28:620-624.

McCabe SE, Cranford JA, Morales M, Young A. 2006. Simultaneous and concurrent poly-drug use of alcohol and prescription drugs: prevalence, correlates and consequences. J Stud Alcohol 67: 529-537.

McCabe SE, Cranford JA, West BT. 2008. Trends in prescription drug abuse and dependence, co-occurrence with other substance use disorders, and treatment utilization: results from two national surveys. Addict Behav 33:1297-1305.

Olière S, Joliette-Riopel A, Potvin S, Jutras-Aswad D. 2013. Modulation of the endocannabinoid system: vulnerability factor and new treatment target for stimulant addiction. Front Psychiatry 4: 1-21.

Olthuis JV, Darredeau C, Barrett SP. 2013. Substance use initiation: the role of simultaneous polysubstance use. Drug Alcohol Rev 32: 67-71.

Rao JNK, Scott AJ. 1984. On chi-squared tests for multi-way tables with cell proportions estimated from survey data. Ann Stat 12: 46-60.

Substance Abuse and Mental Health Services Administration. 2013a. Drug Abuse Warning Network, 2011: National Estimates of Drug-related Emergency Department Visits. HHS Publication No. (SMA) 13-4760, DAWN Series D-39. Center for Behavioral Health Statistics and Quality, Rockville, MD.

Substance Abuse and Mental Health Services Administration. 2013b. The DAWN Report: Emergency Department Visits Involving Attention Deficit/Hyperactivity Disorder Stimulant Medications. Center for Behavioral Health Statistics and Quality, Rockville, MD.

Watson WA, Litovitz TL, Klein-Schwartz W, et al. 2004. 2003 annual report of the American Association of Poison Control Centers Toxic Exposure Surveillance System. Am J Emerg Med 22: 335-404. 